\title{
Jack Russell Terrier, Wire
}

National Cancer Institute

\section{Source}

National Cancer Institute. Jack Russell Terrier, Wire. NCI Thesaurus. Code C53722.

The Jack Russell Terrier Wire is a wire-coated variety of the Jack Russell Terrier. 